\title{
Firenze, Biblioteca Medicea Laurenziana, Plut. 28.13
}

Costantinopoli, terzo quarto del XIV sec.

ff. I-III, 247, IV-V (i fogli di guardia non sono numerati); foliotazione doppia: quella più antica, nel marg. sup. est., è visibile solo su alcuni fogli (ff. 20, 90, 91, 237, 246); quella più recente, cui si fa riferimento, è presente nel marg. inf. est.; sono bianchi i ff. 19v e 98v; cart.; fil. ff. 11, 26, 32, 37, 38, 42, 46, 49, 51, 54, 57, 60, 62, 65, 67, 69, 71, 73, 79 simile a horn (P VII, nr. II/93, 1384), ff. 82, 84, 87, 89 simile a cercle (Br nr. 2939/2940, 1350/1380), ff. 123, 133, 135, 136, 140, 141, 143, 145, 148, 150, 151, 153, 158, 161, 164, 172 , 177, 182, 187 simile a cercle (Br nrr. 3161/3163, 1375/1380), ff. 190, 195, 198, 200, 201, 206, 207, 210, 213, 216, 217, 220, 221, 224, 225, 228, 230, 232, 233, 235, 237, 242, 244, 245, 247 simile a clefs (Br nr. 3847, 1368); $1^{10}(10), 2^{6+1}(17), 3-11^{8}(89), 12^{8+1}(98), 13^{6+1}(105)$, $14^{8+1}(114), 15-30^{8}(242), 31^{4+1}(247)$; due fascicolazioni: la prima riguarda i primi 12 fascicoli, numerati, dal secondo $\left(\beta^{\prime}\right)$ al dodicesimo $\left(\iota \beta^{\prime}\right)$, nel marg. inf. est. del verso del primo foglio di ogni fascicolo, da una mano diversa da quella del copista; la seconda, di mano del copista, è presente nel marg. sup. est. del recto del primo foglio di ogni fascicolo a partire dal f. 99 , ossia dall'attuale fascicolo $13^{\circ}$, segnato come $\alpha^{\prime}$, e procede fino alla fine; $\mathrm{mm} 218 \times 141=15 / / \mathbf{1 7 7} / / 24 \times 17 / / 105 / / 21$ (f. 14), $220 \times 148=20 / / 170 / /$ $30 \times 16$ // 99 // 32 (f. 104); il copista sembra aver tracciato unicamente la linea verticale esterna di giustificazione sul recto di ogni foglio; 11. 34-36; unità di rigatura mm 5; non vi è traccia di foratura. Legatura medicea con catena (sec. XVI).

\section{Scrittura}

Il codice è vergato da una sola mano: <Isacco Argiro (Mondrain 2012, 630)> (f. 1r-19r, 20r-98r, 99r-247r).

\section{Contenuto}

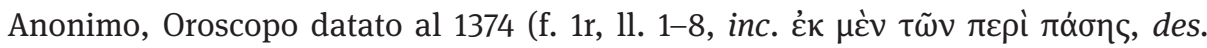

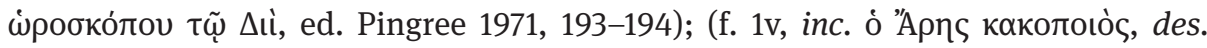

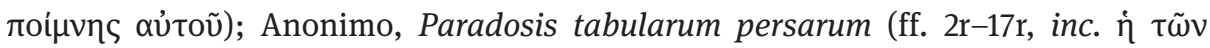

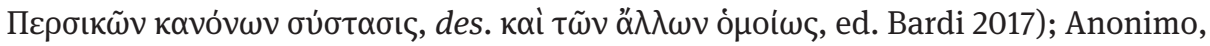

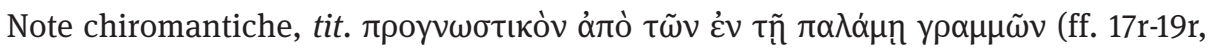

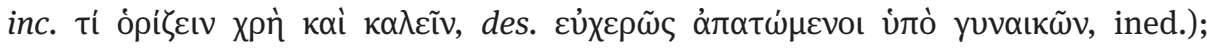
Anonimo, Tabulae persicae (ff. 20r-90v, ined., cf. Tihon 1987); Isacco Argiro, De cyclis

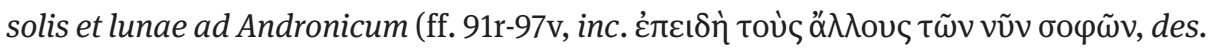

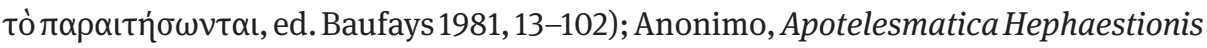




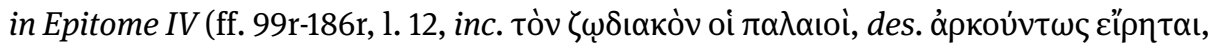
ed. Pingree 1974, 135, 1. 1-347, 1. 99); Anonimo, Notae astrologicae variae (f. 186r, 11.

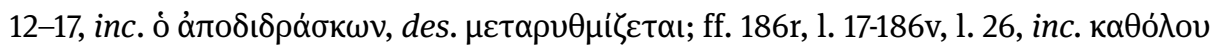

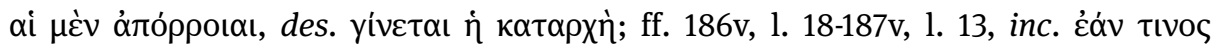

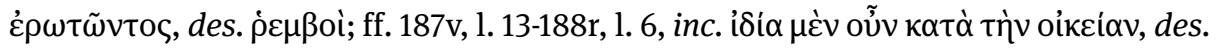

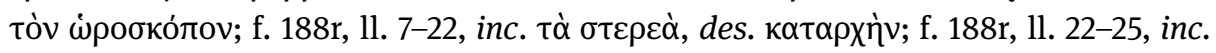

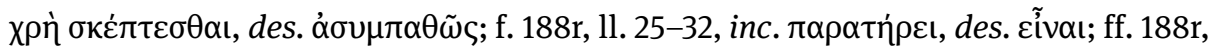

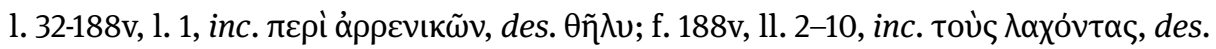

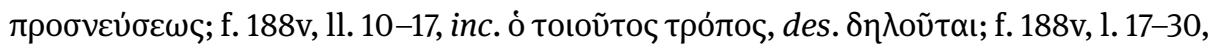

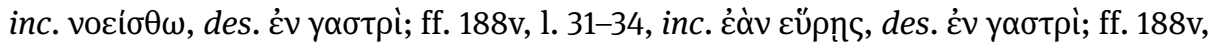

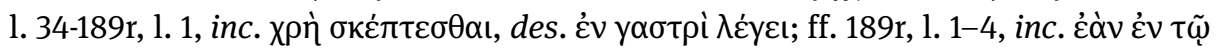

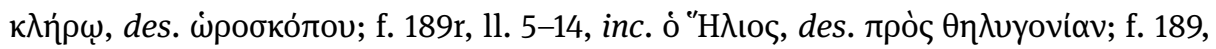

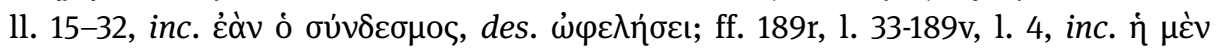

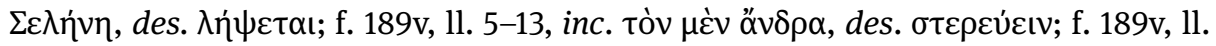

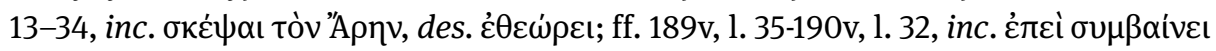

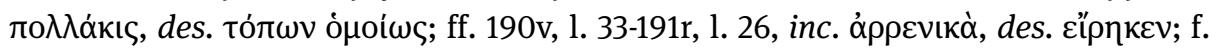

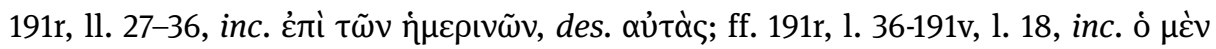

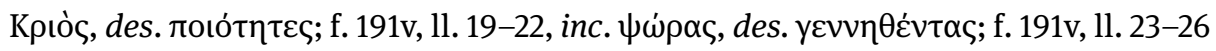

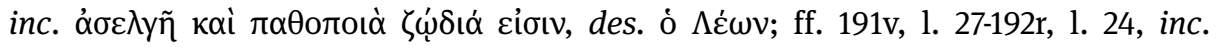

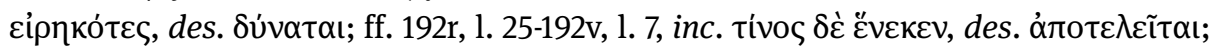

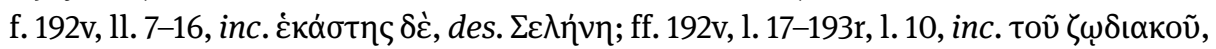

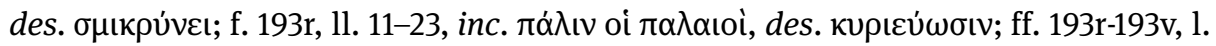

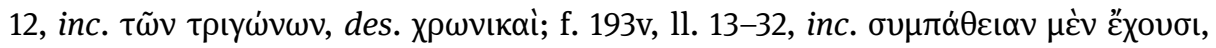

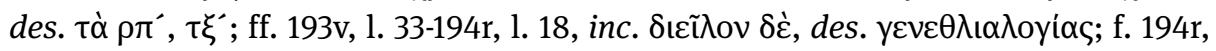

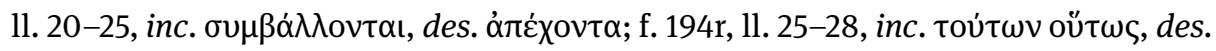

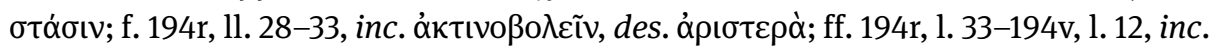

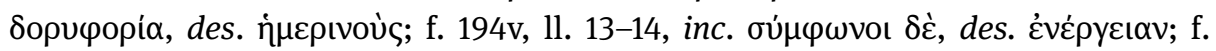

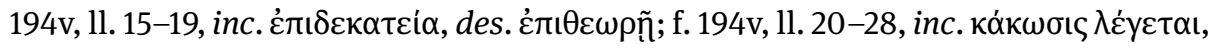

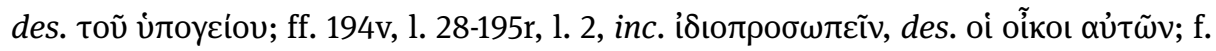

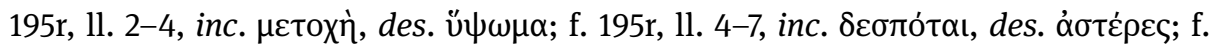

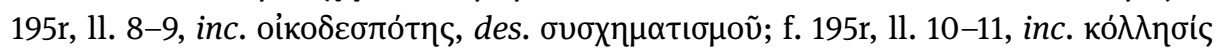

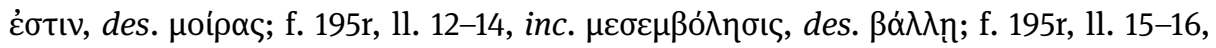

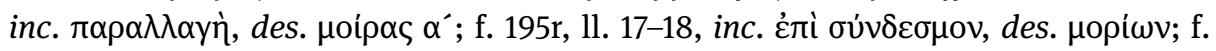

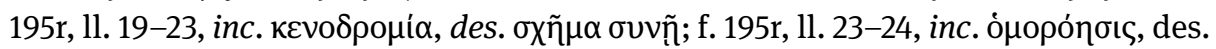

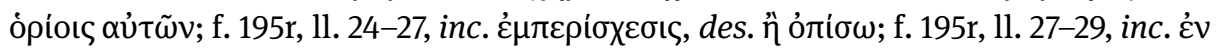

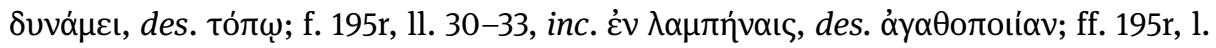

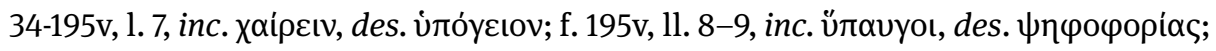

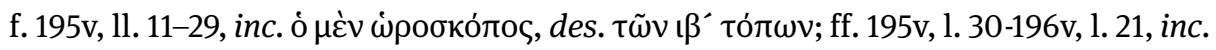

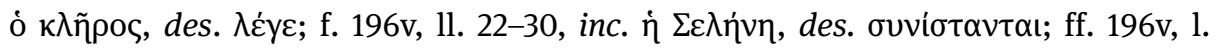

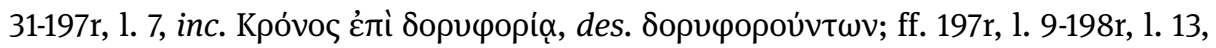

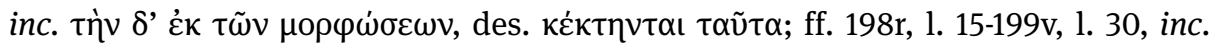

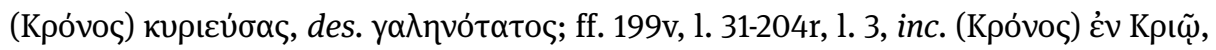




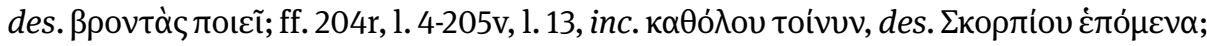

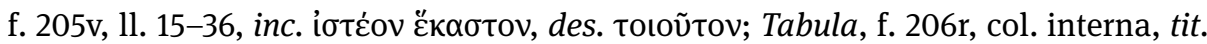

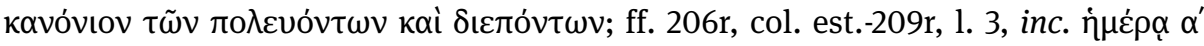

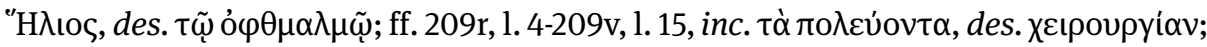

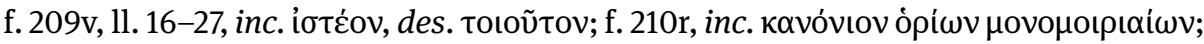

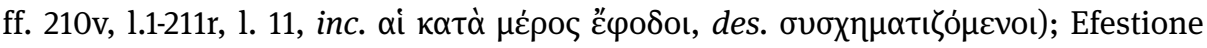

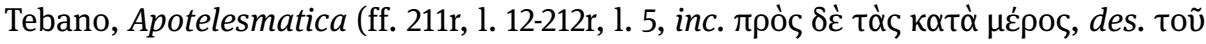
бколои̃, ed. Pingree 1973, 76, 1. 1-80, 11 [testo di L]); Ammonio, Excerpta ex anecdotis

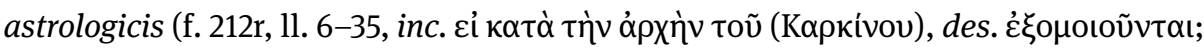

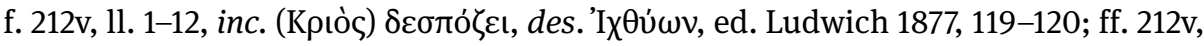

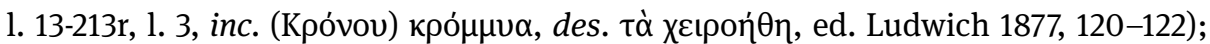
Efestione Tebano, Excerptum ex Apotelesmaticis (ff. 213r, 1. 4-213v, 1. 22, inc. (Kpıoũ)

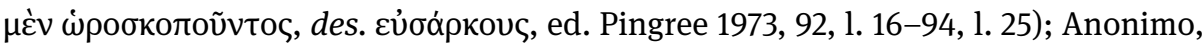
Excerpta (ff. 213v, 1. 23-214r, 1. 25, inc. ả

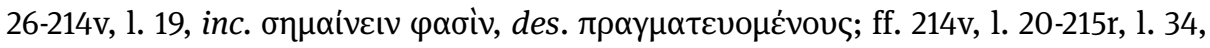

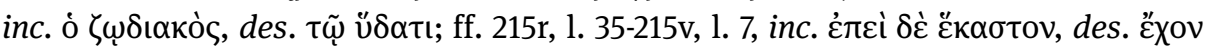

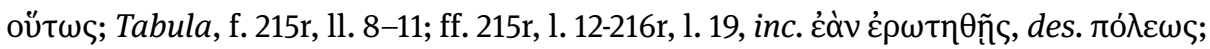
f. 216r, ll. 19-32, inc. غ̇лıтท́

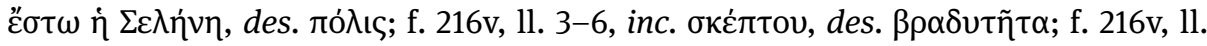

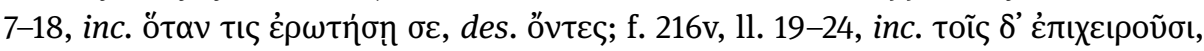

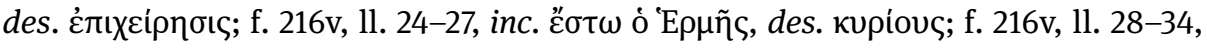

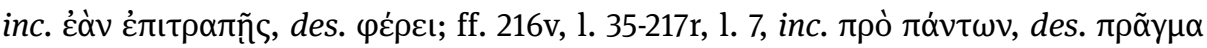

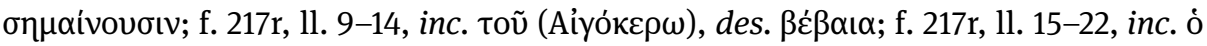

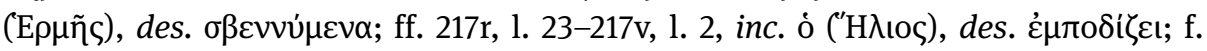

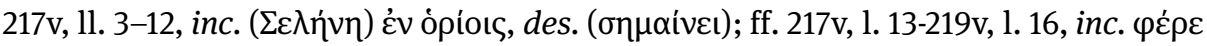

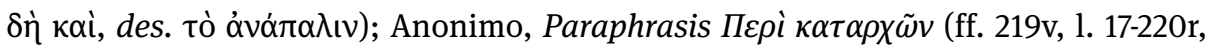

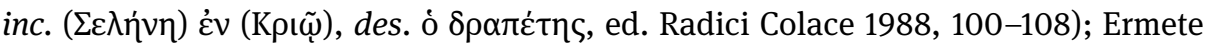

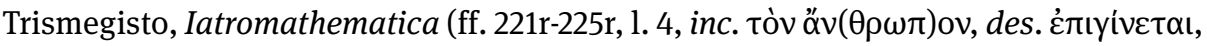
ed. Ideler 1963, 387-396); Galeno, Prognostica de cubito ex mathematica scientia (ff.

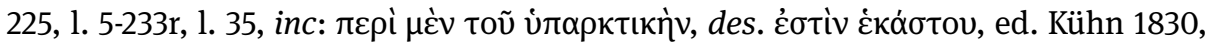

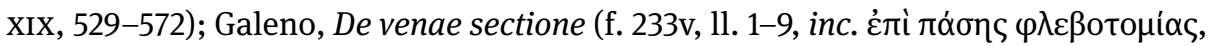
des. ká $\mu v o v \tau ı$, ed. Kühn 1830, 519); Doroteo di Sidone, Fragmenta graeca e Carmine

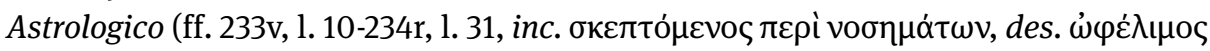
yívetal, ed. Pingree 1976, 422, 1. 10-425, 1. 22); Anonimo, Nota astrologica (ff. 234r, l.

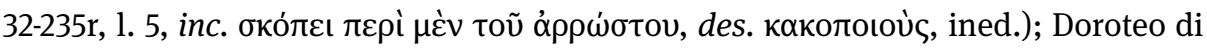
Sidone, Fragmenta graeca e Carmine Astrologico (ff. 235r, 1. 6-236r, 1. 33, inc. ò $\mu \varepsilon \dot{v} v$

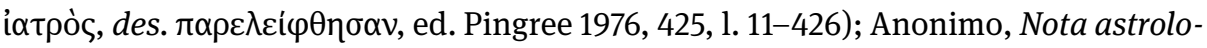

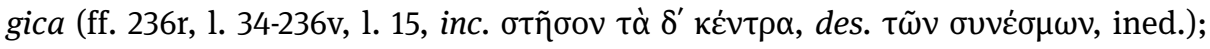
Doroteo di Sidone, Fragmenta graeca e Carmine Astrologico (ff. 236v, 1. 16-237r, 1. 9,

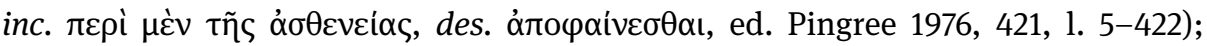

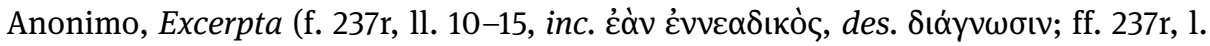

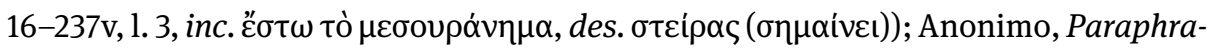




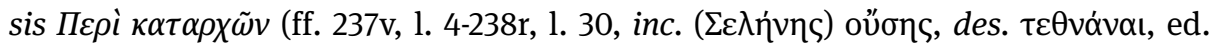
Radici Colace 1988, 86-94); Anonimo, Nota astrologica (ff. 238r, 1. 31-238v, 1.1, inc.

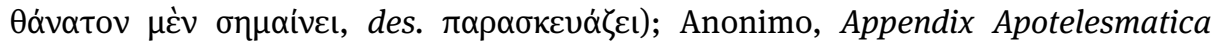

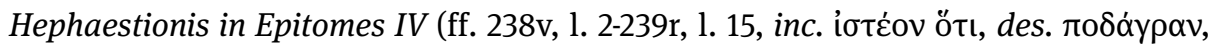

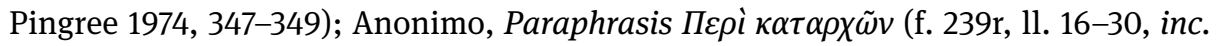

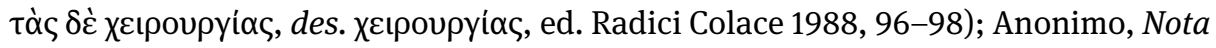

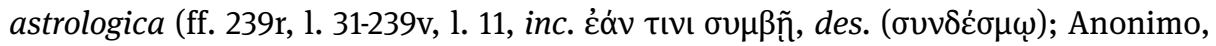
Appendix Apotelesmatica Hephaestionis in Epitomes IV (f. 239v, 1l. 13-27, inc.

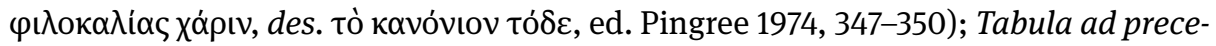
dens pertinens (f. 239v); Stefano Alessandrino, Commentarium (ff. 240r-247v, inc. Toĩs

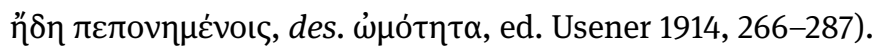

\section{Relazioni stemmatiche}

Il Laur. Plut. 28.13 reca materiale relativo alle cosiddette Tabulae Persicae, seguiti da una lunga serie di excerpta tratti da diverse opere di argomento astrologico. Argiro non fu semplicemente il copista della raccolta, ma anche il suo concepteur: egli ha curato la copia dell'esemplare, la selezione dei testi e la loro organizzazione. Sono copia di questo codice le miscellanee Laur. Plut. 28.16, vergato nel 1382 da Giovanni Abramio, e Laur. Plut. 28.34 (Pingree 1974, Xv).

\section{Storia del codice}

Il codice, passato tra i libri di Crisolora come si deduce dal titolo bilingue vergato al f. 247r (Cataldi Palau 2005, 403), entrò a far parte della biblioteca medicea privata (nr. 146), probabilmente in seguito al viaggio compiuto da Giano Lascari in Grecia nel 1491 per conto di Lorenzo de' Medici. Sembra possibile infatti, se non altro sulla base dell'identità di contenuto, che si tratti dello stesso codice che Lascari vide in casa di Demetrio Trivoli ad Arta. Una volta entrato nella biblioteca medicea, il codice fu visionato e chiesto in prestito il 2 ottobre del 1493 da Pico della Mirandola (Gentile 1994, 94).

\section{Bibliografia}

\section{Cataloghi}

Bandini (1770) II, 25-29; CCAG I, 6-20, nr. 7. 


\section{Edizioni}

Baufays (1981); Ideler (1963); Kühn (1830); Ludwich (1877); Pingree (1971); Pingree (1973); Pingree (1974); Pingree (1976); Radici Colace (1988); Usener (1914).

\section{Codicologia e paleografia}

Cataldi Palau (2005) 402-403; Jackson (1998) 103-104; Gentile (1994) 93-94, scheda nr. 26; Mondrain (2012) 630; Rigo (2002) 73; Speranzi (2008) 221, n. 67.

\section{Studi}

Pingree (1971) 189-194; Radici Colace (1984) 144 e n. 40; Radici Colace (1985) 78-85; Tihon (1987) 471-487; Touwaide (2009) 453-595.

\section{Riproduzioni}

Cataldi Palau (2005), tav. XIX.2. 